\title{
Semantic trajectory compression: Representing urban movement in a nutshell*
}

\author{
Kai-Florian Richter ${ }^{1,2}$, Falko Schmid ${ }^{1}$, and Patrick Laube ${ }^{3}$ \\ ${ }^{1}$ SFB / TR 8 Spatial Cognition, University of Bremen, Germany \\ ${ }^{2}$ Department of Infrastructure Engineering, University of Melbourne, Australia \\ ${ }^{3}$ Department of Geography, University of Zurich, Switzerland
}

Received: June 06, 2011; returned: September 07, 2011; revised: December 22, 2011; accepted: May 05, 2012.

\begin{abstract}
There is an increasing number of rapidly growing repositories capturing the movement of people in space-time. Movement trajectory compression becomes an obvious necessity for coping with such growing data volumes. This paper introduces the concept of semantic trajectory compression (STC). STC allows for substantially compressing trajectory data with acceptable information loss. It exploits that human urban mobility typically occurs in transportation networks that define a geographic context for the movement. In STC, a semantic representation of the trajectory that consists of reference points localized in a transportation network replaces raw, highly redundant position information (e.g., from GPS receivers). An experimental evaluation with real and synthetic trajectories demonstrates the power of STC in reducing trajectories to essential information and illustrates how trajectories can be restored from compressed data. The paper discusses possible application areas of STC trajectories.
\end{abstract}

Keywords: trajectories, moving objects, semantic description, data compression, transportation network, chunking, navigation, map matching

\section{Introduction}

Tracking devices mechanistically capture individual movement as trajectories that consist of a series of positioning fixes. Consecutive fixes are co-located, in the sense that temporally neighboring fixes refer to similar positions in space. As a result, trajectory data

\footnotetext{
${ }^{*}$ This full paper revises and extends an extended abstract version presented at the poster session of the 11th International Symposium on Spatial and Temporal Databases, July 8-10, 2009, Aalborg, Denmark |39|.
} 
is highly spatiotemporally autocorrelated and can be considered redundant. By contrast, humans plan, perceive, and communicate journeys as legs traveled in geographic space, typically following a street network or using train or bus lines. Especially in urban environments there are few alternatives to following available transportation infrastructure network links. This paper exploits such semantic grounding of urban movement for compressing trajectory data. In doing so, the paper addresses the GIScience priority of bridging the "gulf between low-level observational data (fixes) and high-level conceptual schemes (journeys) through which we as humans interpret, understand, and use that data" ( [16], p. $300)$.

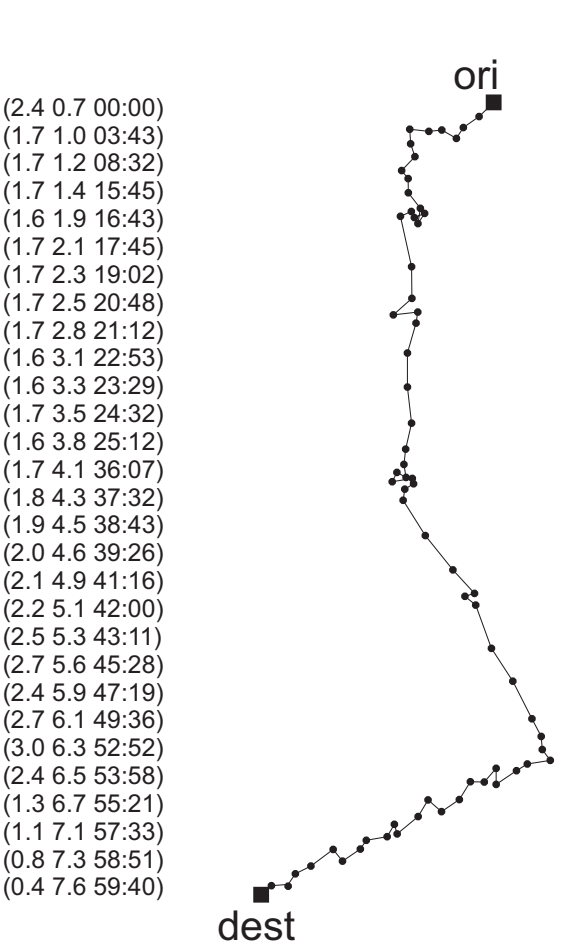

(a) (b)

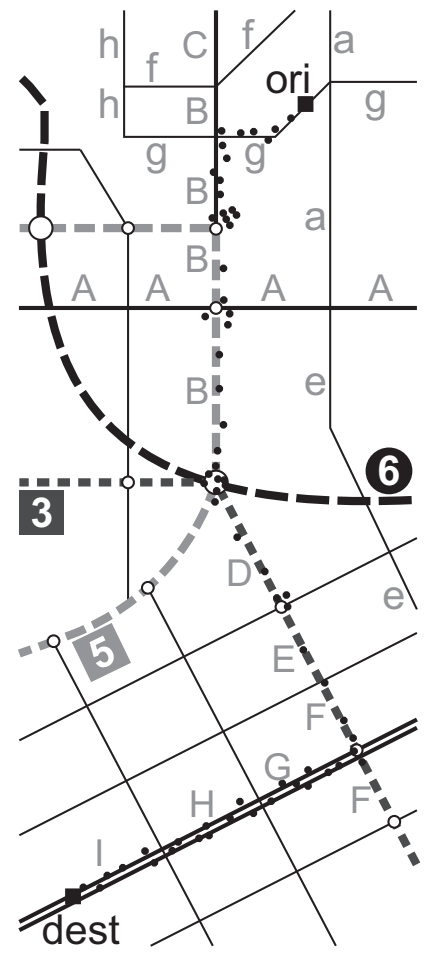

(c)

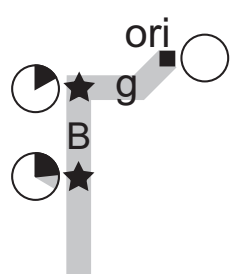

5

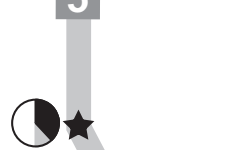

3

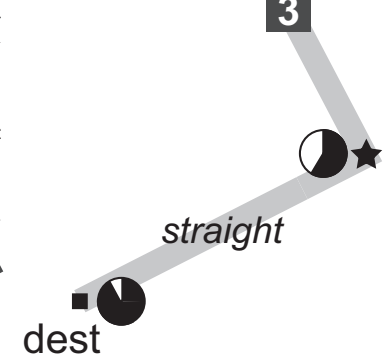

(d)

Figure 1: Problem overview (adapted from |39|). (a) Raw positional data as fixes $(x, y, t$ tuples); (b) trajectory in two-dimensional space, object moving from origin to destination; (c) trajectory embedded in semantic geographic context; (d) compressed representation of the same trajectory with timestamped reference points as indicated by the clock symbols: from origin, to street $g$, street $B$, tram line $\# 5$, tram line $\# 3$, straight, to destination, clock symbols with grey sectors represent intervals.

Consider the trajectory illustrated in Figure 1 which captures the movement of a commuter from home to work. An array of fixes produced by a tracking system (e.g., GPS) is listed in Figure 1a and mapped as a trajectory in Figure 1 2 . Figure 1 1 depicts the same 
movement embedded in its geographical context. The commuter followed an urban transportation network rather than moving in an unconstrained space.

This example illustrates that the observed movement can be modeled by referring to elements of the network semantics. The course can be expressed by the streets and tram tracks it moves along (street $g$, street $B$, tram line $\# 5$, tram line \#3) or by references to the movement pattern on the network (straight, to destination). The temporal aspect of the trajectory is captured in the respective timestamps. Figure $1 \mathrm{~d}$ illustrates this minimalist representation of the observed movement. The use of semantics for trajectory compression exploits related research in the field of spatial cognition (which is concerned with the acquisition, organization, utilization, and revision of knowledge about spatial environments) that aims to design better wayfinding instructions |10, $24.45 \mid$.

This paper brings together the reduction of spatial dimensionality of networkconstrained movement, the data aggregation potential of semantic information captured in the geographic context of movement, and the theory of wayfinding from spatial cognition. It proposes a novel approach for compressing trajectory data. In detail, the paper advances geographical information science with the following contributions:

- the concept of semantic trajectory compression (STC), exploiting the semantic embedding of movement for deflating highly redundant trajectory data for an efficient management of movement data;

- the algorithm STC, integrating and extending methods from navigation research (map matching, place identification) and spatial cognition (wayfinding, generation of directions); and

- an evaluation of STC based on a real, example tracking dataset, which has been recorded by GPS in an urban context, as well as synthetic trajectories that have been generated to further explore properties of STC.

STC relies on the availability of transport network data for compression. While this does not cover every type of human movement through space (e.g., hiking in open space national parks), many of today's trajectory repositories cover movement of (commercial) vehicles or pedestrian movement in urban areas (e.g., motorcycle courier trajectories in London ${ }^{1}$, schoolbus and truck trajectories in the Athens metropolitan area ${ }^{2}$, or taxi trajectories in Beijing, as part of the GeoLife GPS data ${ }^{3}$ ).

This article is a full paper version of [39|, presented as an extended abstract at the poster session of the 11th International Symposium on Spatial and Temporal Databases, July 810, 2009, Aalborg, Denmark. This full paper presents original work, including a related work section, an elaborated framework of semantics in networks, a detailed description of the proposed algorithms, extensive experimental evaluation sections, and a section on applications of STC trajectories.

\section{Related work}

Recent work in the areas of database research, spatial cognition, and geographical information science is particularly relevant to the development of STC. This section provides an

\footnotetext{
${ }^{1}$ http://api.ecourier.co.uk

${ }^{2}$ http://www.rtreeportal.org

${ }^{3}$ http://research.microsoft.com/en-us/ downloads / b16d359d-d164-469e-9fd4-daa38f2b2e13 /
} 
overview and synthesis of the following four topics: previous work on path and line simplification; the notion and use of the semantic embedding of trajectories in their geographical context; the use of such semantic information for enriching trajectory data; and finally, previous work on modeling and querying moving objects in moving object databases (MOD).

Path and line simplification As a reaction to ever increasing volumes of spatiotemporal data, recent years have seen initial work on techniques for the compression of such data. With respect to trajectories, the vast toolbox for line generalization appears to offer a quick fix for trajectory compression (e.g., |12|). However, Meratnia and de By [30| argue that conventional spatial line generalization techniques are not suited for spatiotemporal trajectory compression as they ignore temporal aspects in determining the information to retain of the trajectories. Consequently, Meratnia and de By propose alternative geometrybased approaches. Moreover, trajectories are inherently spatiotemporal and compression algorithms should preserve both their spatial and temporal properties. Modeling time as a third spatial dimension, Cao et al. |7| and Gudmundsson et al. $|19|-$ presenting an approximate version of the Douglas-Peucker algorithm - propose path simplification algorithms for three-dimensional spaces $(x, y, t)$. Path-simplification is mostly concerned with the question "When is a line segment acceptable as an approximation for a subpath?" which is often tested with some form of tolerance band $\varepsilon$. Additional to shape-preserving algorithms, Potamias et al. [32| focus on preserving spatiotemporal features inherent in the trajectory (such as speed or orientation) when subsampling. Both computational geometry and database communities aim at compression techniques that still allow queries (e.g., where at, when at, nearest neighbor) to be answered once the trajectory has been compressed. In general, path-simplification is geometry-driven, be it 2D or 3D. Recently, alternative strategies are discussed that exploit the semantics of trajectories, that is the meaning of a movement path to the moving actors and its embedding in the underlying geography.

Network bound movement The majority of movement planned or performed by humans is bound to some form of network, be it an urban street network, a rail network, or even an air traffic network. In recent years, this a priori knowledge is increasingly exploited for the handling of large volumes of trajectory data.

Linking GPS fixes to a network requires map matching, the process of matching a position datum with a map, i.e., identifying the corresponding infrastructure elements for coordinates in a geographic data set. Mostly, GPS coordinates are matched against street data but there are indoor map matching algorithms as well (e.g., for robot navigation). The increasing ubiquity of GPS sensors in vehicles and mobile devices makes it a vivid research area that aims at improving the accuracy of element identification. The simplest form of map matching is a point-to-point approach: a position datum is matched against a vertex of a street network. Point-to-curve algorithms match the estimated position against edges of the network. In street networks they usually provide a higher accuracy, as vertices may be comparably widely spread (see $[4.46 \mid$ for an overview of these approaches). More sophisticated algorithms consider topological information of the network, traveling constraints introduced by transportation means, and heading information. These algorithms outperform the simple approaches as they can detect unlikely and impossible network assignments [5:6.18.33.34].

Once trajectories are mapped to a network, the spatial dimensionality is reduced, which allows for efficient indexing structures for moving objects [27]. For example, Tiesyte and 
Jensen [43| exploit a main-memory index structure enabling incremental similarity search for trajectories of vehicles moving on known routes. The key benefit of such reference systems lies in their reduced spatial dimensionality (convoluted arcs are reduced to linear edges), which enable more efficient indexing.

A priori knowledge about objects moving in a network can then further be used for compressing trajectories. For example, Cao and Wolfson $|6|$ model movement along a directed graph that consists of nodes representing intersections and connecting edges representing road segments. Instead of storing large amounts of locational fixes, their non-materialized trajectories model movement as sequences of tuples (on street $p_{i}$, at location $l_{i}$ along $p_{i}$, at time $t_{i}$ ). Since tuples on the same segment can be merged, non-materialized trajectories require less storage data than raw trajectory data.

Exploiting the semantics of movement Going one step further beyond just linking movement to a network graph, the embedding of movement in the underlying geography offers further a priori knowledge exploitable for trajectory compression.

When compressing trajectories beyond pure geometry, activities (e.g., stops) along the traveled paths need to be preserved. Several algorithmic approaches identify such activities (called places from here on) within GPS data. Differences in approaches usually stem from the intended application. There are algorithms for detecting place concepts for human behavior learning [29], for diary applications [8], or for wayfinding assistance [38]. However, all of them consider places to be spatiotemporal clusters, i.e., accumulations of position fixes in a certain region of a certain size. Most algorithms rely on explicit values for the two crucial parameters time and region size (e.g., 10 minutes, 50 square meters) that define the granularity of the detected places. Some algorithms are parameter-free [38|, but detect places on a finer resolution which then are post-processed and selected according to their long-term significance. For trajectory compression the prime interest is in detecting significant reference points and not in generating familiarity profiles. STC relies on a simpler fixed parameter algorithm |3 23|.

The database community initially focused mainly on the definition of spatiotemporal data types (e.g., moving point and moving region data types [22 47]). Such spatiotemporal data types allow for queries such as "Find cars that stopped today at point $\langle x, y\rangle$ at time $\left.\langle t\rangle^{\prime \prime}\right)$. However, queries referring to the semantic meaning of the movement, such as "Find cars which stopped today at the gas station at time $\langle t\rangle^{\prime \prime}$ cannot easily be answered [47| because this requires data beyond the trajectory, namely a matching between point $\langle x, y\rangle$ and the geographic feature "the gas station." For that reason, more recent research builds on conceptual models of movement, exploring techniques for adding semantic information to trajectories in order to facilitate trajectory analysis in different application domains [2].

Semantic trajectory enriching requires application domain knowledge, as the user must specify what spatial features are relevant to the analysis of trajectories [2.42] (e.g., hotels, touristic places, or stop-over locations of migrating birds). In STC, the context is always the same and, hence, the characterization of reference points can be done in a much more deterministic way.

Querying moving objects Since the late nineties moving object databases (MODs) developed as a special branch of spatiotemporal databases [22|. The key challenge for MODs lies in keeping track of the constantly changing location of moving objects, balancing positional inaccuracy and costly updates [21]. This section reviews several dimensions of queries for 
moving objects, allowing a final discussion about querying STC compressed trajectories (see Section 7).

Location-based queries are a first type of moving object query [1], including nearestneighbor queries (NN, "Given a moving object $O_{1}$ and an interval $i$, which other moving object $O_{x}$ is closest to $O_{1}$ during $i$ ?") and range queries ("Given a rectangle $R$ and a time $t$, which moving objects $O_{x}$ are within $R$ at $t$ ?"). The second type, trajectory queries, refer to the trajectory as a timestamped polyline, including similarity queries ("Which trajectories are similar in shape?") and parameter queries ("Which trajectories move north?").

MODs model and query past as well as present and near future movement. The latter is especially challenging as it includes the notion of forecasting and querying future positions. The MOST data model (moving-objects spatiotemporal) and the corresponding FTL (future temporal logic) allow querying present and future states through the use of dynamic attributes, extended data types that change implicitly over time [22|. MODs consequently feature various query modes, producing different results as time progresses [40|. Instantaneous queries are evaluated once, at time $t$ ("Return the bus stops within $2 \mathrm{~km}$ from my current position"). Continuous queries "travel" with the moving object and are a sequence of instantaneous queries re-evaluated for all $t^{\prime}>t$ ("Return the bus stops within $2 \mathrm{~km}$ from my current (and constantly updated) position"). Finally, a persistent query at time $t$ is a sequence of instantaneous queries on the infinite history starting at $t$ ("Retrieve the objects whose speed doubles within 10 minutes").

Most research on MODs considers movement in an unconstrained Euclidean space. Only recently, the MOD community addressed network-bound movement [21 31]. Especially relevant for this paper is the work of [31], as the authors adopt $N N$, range, and closest-pair ("Find the hotel-restaurant pair within the smallest driving distance.") queries for spatial network databases.

\section{Trajectory and network semantics}

Most tracking systems capture the movement of individuals as trajectories. They store lists of timestamped position samples, so-called fixes, in the form of tuples $(x, y, t)$. In its most simple form a trajectory is a polygonal line connecting the fixes of a moving individual |20|. Formally, the trajectory $T$ of a moving object $O$ over time interval $\left[t_{i}, t_{j}\right]$ is described by

$$
T_{O}(i, j)=\left\langle\left(x_{i}, y_{i}, t_{i}\right),\left(x_{i+1}, y_{i+1}, t_{i+1}\right), \ldots,\left(x_{j}, y_{j}, t_{j}\right)\right\rangle .
$$

$T_{O}$ is linear on each interval $\left[t_{i}, t_{i+1}\right]$.

The example trajectory in Figure 1 shows an object moving from origin ori to destination dest, over a temporal interval from 00:00 to 59:40. Figure 1a lists the fixes of the trajectory, Figure 10 the respective trajectory mapped in two-dimensional space. Note that only a subset of all fixes are listed in Figure 1a.

A map is a semantically annotated network of edges and nodes. A map represents an urban transportation network, featuring streets, bus, tram, and train lines (see Figure 1:). A map has the following properties:

- Vertices are unambiguously defined, either by IDs or by $(x, y)$ coordinate tuples.

- Edges have labels (street name; or bus, tram, train line, e.g., $B$ or 3 in Figure 1:).

www.josis.org 
- Bus, tram, and train lines can (but need not) be superimposed upon the street network. Vertices of bus, tram, and train lines are stops and stations; these may be labeled with the stops' names.

- The labeling of edges and vertices can extend several levels of granularity. An edge may at the same time have a local street name (e.g., "Ostertorsteinweg") and be part of a national highway system (e.g., "A7").

Formally, a map is described by:

$$
M=\left(V_{T}, E_{T}, V_{G}, E_{G}\right)
$$

$V_{T}$ is a set of topological vertices. These are the intersections of a transport network that define the links between the other topological entities. $E_{T}$ is the set of topological edges between the elements of $V_{T}$. Most path planning algorithms work on the elements of $V_{T}$ and $E_{T}$, which is termed the network graph. This is also the graph compression and decompression of trajectories mostly work on. For some operations, though, these processes need to use the geometry of the network configuration. This geometry is described by $V_{G}, E_{G}$, the sets of geometric vertices and edges. With these sets the actual layout of spatial entities in the environment is described. Map matching works on the geometric representation of streets (although sophisticated approaches use the topological representation in parallel), and turning angles, i.e., angles between pairs of streets, are determined on this level. For each topological edge there is a corresponding sequence of geometric edges. Topology abstracts from geometry to connectedness. Figure 2 illustrates this. In Figure 2 the topological edge $e_{T 1 T 2}$, which connects the topological vertices $v_{T 1}$ and $v_{T 2}$, abstracts from the sequence of five geometric edges $e_{G 1}$ to $e_{G 5}$ that describe the course between the geometric vertices $v_{G 1}$ and $v_{G 2}$. Note that topological vertices always coincide with geometric vertices (the former are a subset of the latter), but topological edges do not need to correspond to geometric edges.

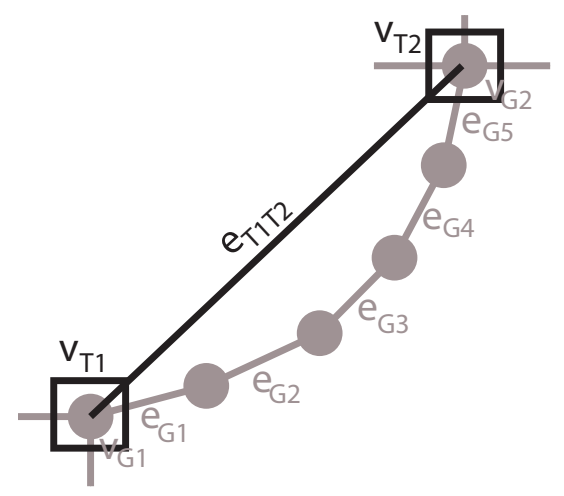

Figure 2: Illustration of how topology abstracts from geometry. The topological edge $e_{T 1 T 2}$ abstracts from the five geometric edges $e_{G 1}$ to $e_{G 5}$.

The advantage of a network lies in reducing the dimensionality of a two-dimensional movement space. An object moving in a network can concisely be positioned along edges and at vertices through timestamping. Given a semantically annotated map, edges and 
vertices can be aggregated according to shared labels. Often, several consecutive edges represent the same street and, thus, share the same label. Tram and bus lines may even extend over large sections of a transport network. In short, STC exploits the high-level reference system that comes with the semantic annotation of the transportation network.

Taking this perspective, streets and tram, bus, or train lines can be viewed as mobility channels that moving objects hop on, ride for a while, and hop off again to catch another channel that brings them closer to their destination. Only small quantities of information need to be stored for adequately representing the movement of an object riding such channels. With respect to data compression, this mobility channel metaphor illustrates the potential for trimming large amounts of redundant fixes. All that needs to be stored to get an accurate approximation of individuals driving, cycling, or walking through a network are the location and hop-on and hop-off times where an object enters or leaves such a channel (e.g., street intersections or bus stops).

Finally, reference points structure trajectories. These reference points are the spatiotemporal location of where the relevant actions describing the movement of an object in a network happen. Reference points are structurally modeled as points on the network annotated with time intervals. The intervals conflate to a time point when changing the mobility channel can be considered instantaneous (e.g., turning from one street into another). STC considers the following types of reference points, which suffice to describe movement in a network:

- Origin and destination of a trajectory clearly are reference points; they define the spatiotemporal boundaries of the represented movement behavior.

- Street intersections and public transport stops are taken as reference points since here channels may be changed.

- Stops within edges constitute reference points. For instance, these stops may occur when a traveler gets stuck in traffic or decides to interrupt the journey to enter a shop. Such stops are identified as places within the trajectory and are subsequently treated as reference points. Their identification is further discussed in the next section.

To summarize, a reference point is a point on the network with an associated time interval whose position corresponds to one of the following semantic elements: origin, destination, street intersection, public transport stop, place.

\section{Semantic trajectory compression}

This section gives a detailed description of the semantic trajectory compression (STC) approach. In the following, the algorithms for compression (Section 4.1) and decompression (Section 4.2 ) of trajectories are explained. Section 5 then presents an experimental evaluation of both steps.

\subsection{Compression}

STC is based on the discussion of network semantics in Section 3 . Beyond addressing annotated network entities, STC follows a semantic approach in how it interprets the trajectory and utilizes this interpretation for compression. STC transforms the geometry of a trajectory into a semantic representation that is inspired by mechanisms employed in giving 
route directions as they are identified in research on human spatial cognition [10.24 45]. Trajectories are described not just through geometry, but by qualitative descriptions of actions along the network. This way, STC can identify and address structures in the environment that are only graspable if considering how a human perceives it while moving through it. These structures then allow for reducing trajectory descriptions to their semantically relevant cores. Broadly, STC is based on three steps (see Figure 3):

1. In a pre-processing step, identify the relevant reference points along the trajectory.

2. For each reference point, determine all possible descriptions of how movement continues from here.

3. Based on the descriptions, combine consecutive reference points into sequences of reference points. These sequences are termed chunks $\mid 24$ 26|. The compressed trajectory consists of sequences of such chunks.

Identification of reference points Next to its spatial layout, the temporal order of its reference points is the most important property of a trajectory. However, not every single datum is important to describe the semantics of network movement. Time and location of changing mobility channels and the places (stops along the way) are sufficient to capture these semantics. In a pre-processing step, map matching identifies the positions of origin, destination, possible channel changes, and places on the network. The spatial part of a mapped trajectory corresponds to a path through the network; all intersections are vertices of $V_{T}$ (see Figure 3, center).

Places are detected by spatiotemporal clustering, similar to the basic accumulative approach described in [23|. A place is detected if the position lies for a defined time in a defined area (e.g., the signal is for at least ten minutes within an area of 50 meters). The place itself is described by the centroid of all measurements, the spatial center of the fixes assigned to the cluster. This centroid is mapped to the closest network entity, which may be an edge or a vertex. It is treated as a single, outstanding reference point. The time interval for the place is computed by the duration of the cluster. Places are special reference points in that, here, the duration of the cluster is significantly longer than for simple channelchanging reference points or stops at traffic lights. Places mark semantically meaningful stops along the way (e.g., for entering buildings), introducing to a topological map new vertices to represent a place's location. Figure 4 illustrates this: Figure 4 a shows a cluster of positioning signals within temporal and spatial boundaries. In Figure $4 \mathrm{t}$ the centroid $c$ is assigned to the closest network entity (faded-out star mapped on the street segment), introducing a new vertex on the edge the centroid is mapped to.

Channel-changing reference points are annotated with a time point. This point (the timestamp) is interpolated from the timestamps of the fix before and the fix after the reference point. Figure $4:$ and $d$ illustrate the interpolation of a channel-changing reference point: the reference point $t_{e}$ is computed from the fixes $t_{1}$ and $t_{2}$, which are before $\left(t_{1}\right)$, respectively after $\left(t_{2}\right)$ the channel change.

A simple point-to-point map matching has a complexity of $O(n \log n)$ if using efficient lookup data structures, such as R-trees or its variants, for finding the points in $\left(V_{G}, E_{G}\right)$ that match with a trajectory fix (note that the lookup data structure has to be built only once for a geographic area in a preprocessing step). Identification of reference points is of linear complexity, and can be integrated into the map-matching process. Interpolation of 


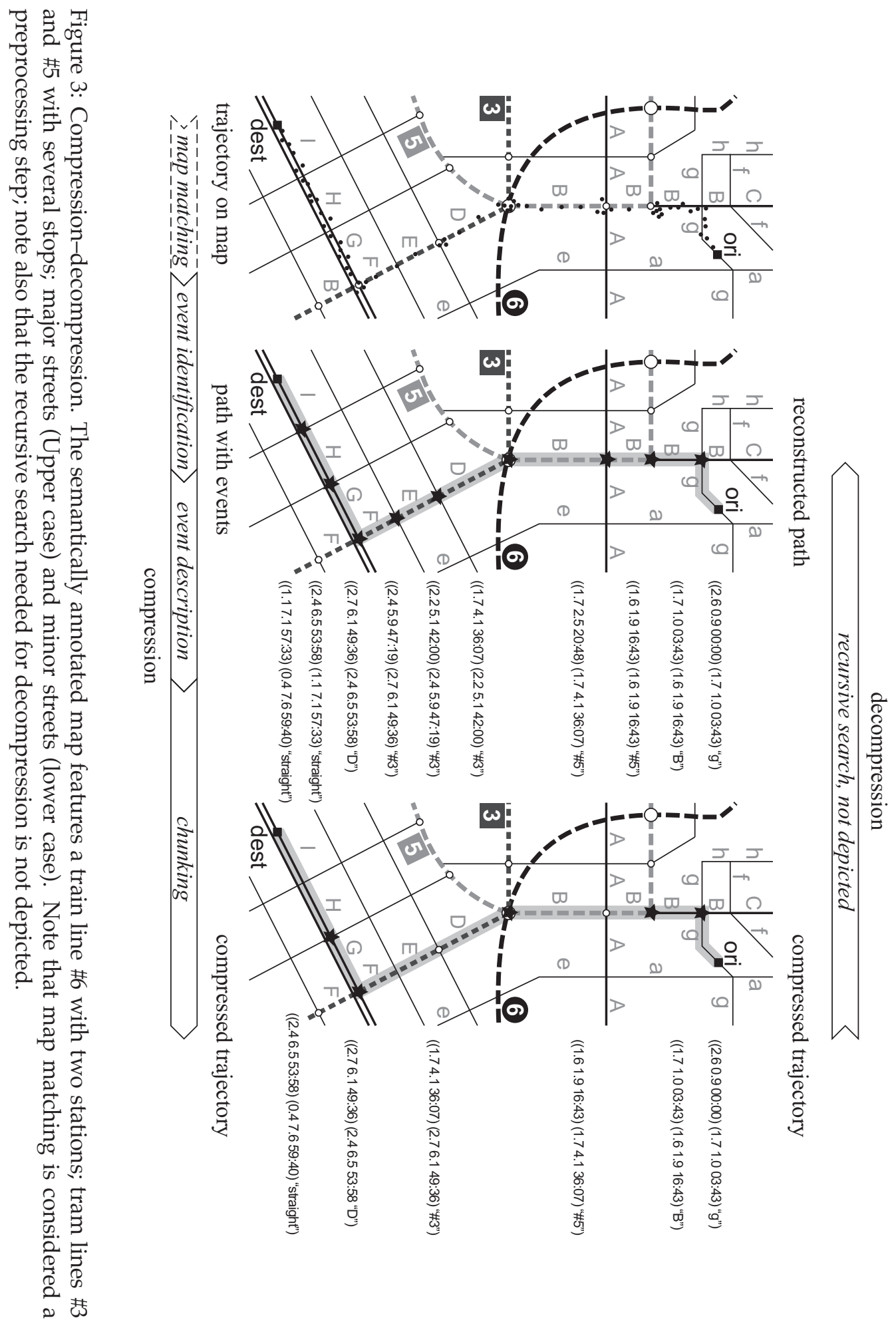


(a)

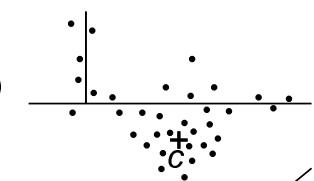

(c)

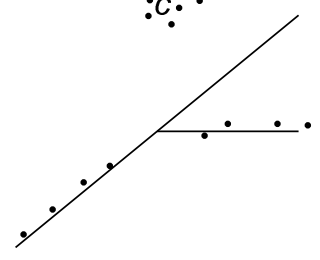

(b)

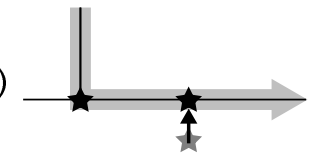

(d)

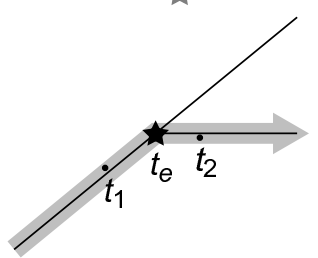

Figure 4: Handling of time. (a) and (b) illustrate the mapping of place centroids against the network infrastructure. (c) and (d) illustrate the interpolation of timestamps for reference points.

timestamps as shown in Figure $4 z$, d requires to iterate the matched trajectory once, as does place identification as proposed by [23], for example.

Description of reference points For each reference point, unambiguous descriptions of how to proceed from here are generated. The semantics of the underlying network determine these descriptions; this makes use of work on cognitively ergonomic route directions [10 35]. Two types of descriptions are used for capturing the motion continuation at a reference point (see Figure 3 center): egocentric direction relation and network labels. Egocentric direction relations express changes of directions in terms of qualitative relations, such as straight, left, or right. The semantics of these relations depend on the underlying qualitative direction model (STC employs the model of [25|). Labels of network elements identify these elements; changes in the labels indicate movement onto the next element. For example, moving from the third $(1.6,1.9)$ to the fourth $(1.72,2.5)$ node in Figure 3 center can be described as straight, $B$, or tram $\# 5$.

In route directions, several consecutive instructions (describing single intersections as exemplified above) can be subsumed into a single, higher-level instruction (e.g., "turn left at the third intersection" instead of "straight, straight, left"; cf. [26|). For their original purpose, these higher-level instructions need to be easily understandable for human wayfinders. Accordingly, the subsumption process needs to make sure that references within and between instructions are unambiguous and comprehensible. This may leave some redundant information in the route descriptions. For example, instructing travelers to go straight for the next 47 intersections imposes a cognitive burden on them that is bound to lead to navigation errors and, thus, this single instruction needs to be broken down to several, more manageable instructions. In STC, these references are not required and, thus, redundancy can be removed (the STC algorithm has no problem with counting to 47). Instead of trying to ease the task of finding a way through an environment, the descriptions are optimized for the purpose of compression.

Compression of reference points Spatial chunking exploits the spatial structure of an environment. It combines descriptions for several consecutive segments of a path into a single higher-level description. Spatial chunking was discussed in depth in [24]; there, a 
formal framework was defined along with an exhaustive set of chunking principles. The applicability of a chunking principle depends on the type of description at hand.

The spatial part of trajectories can be compressed using spatial chunking. The descriptions of movement between reference points used in STC (egocentric directions and network labels) require numerical chunking as the chunking principle to use. In numerical chunking chunks combine consecutive reference points as long as the same description is used for them (e.g., "tram line \#3" chunk in Figure 3). Put differently, reference points are joined until there is a change in description. For example, as long as a trajectory follows edges with identical labels, all these edges are combined into a single chunk by this label. As soon as the label changes, a new chunk is generated. The aim in compression is to create the minimal number of sequences that describe the spatial footprint of a complete trajectory, i.e., the minimal number of chunks. As a consequence, most chunks can be expected to cover a considerable part of the trajectory. Generating this sequence is realized as an optimization problem.

Chunking results in a semantically compact description of a trajectory. The chunks reflect parts of the trajectory where no semantic changes occur, i.e., where an individual moved in a coherent part of the network. The crucial information that needs to be stored in the compressed trajectory is where and when a chunk starts and ends. Furthermore, any subsequent restoration of the path exploits the description the chunk is based on. Each chunk is stored as a tuple (start,description). Element start is a vertex of $V_{G}$ with a time interval, i.e., a $(x, y, t)$ tuple. Element description is stored as an ID value that is used to identify elements of the network in a lookup table (e.g., a hash table). Since every chunk ends at the start-element of its successor, there is no need to store an end-element in a chunk, unless for the last chunk, which also contains the timestamped position of the destination, i.e., where the described movement has stopped. Note that this is a lossy compression with respect to time, since only for those reference points explicitly stored in the compressed trajectory their time interval is retained. It is also lossy in geometry, as it matches individual fixes to vertices and edges of the network.

Algorithm 1 summarizes steps 2 and 3 of the semantic trajectory compression process. Function get_all_actions returns all possible descriptions for a single reference point, function chunk finds the optimal sequence of chunks for the list of reference points (cf. [35|). Function get_all_actions performs two operations for each reference point. It returns the set of labels of the outgoing edge (the network labels) and it calculates the egocentric direction relation between incoming and outgoing edge, i.e., an angle that is converted to a relation symbol. The union of the label set and the relation symbol represents all possible descriptions for the reference point at hand.

As discussed in $[35 \mid$, computational complexity of optimization is dominated by the number of reference points $n$. Richter [35| introduced two different optimization methods: local and global optimization. Global optimization is guaranteed to find the optimum, but has exponential complexity of $O\left(2^{n-1}\right)$. Local optimization has quadratic complexity, $O\left(n^{2}\right)$. While it is not guaranteed to find the optimal solution, extensive tests showed that this happens less than $1 \%$ of the time, and that in these cases the sub-optimal solution is only marginally longer [35|. Thus, the function chunk is implemented using local optimization, with computational complexity of $O\left(n^{2}\right)$. As this is the dominating function in the compression algorithm, this is also the overall complexity of STC. 


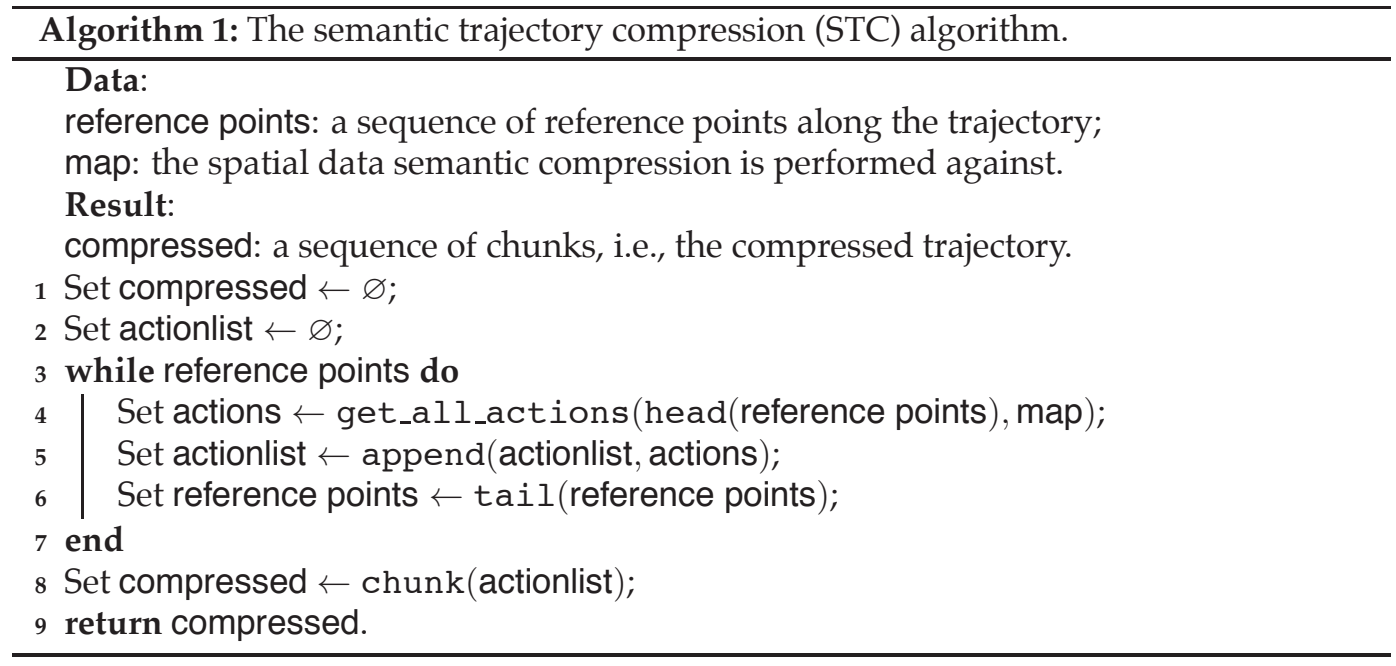

\subsection{Decompression}

In decompression, the aim is to reconstruct movement through an environment. As discussed before, in the chosen semantic approach, decompression does not restore the original trajectory, but rather the reference points that define this movement (see Figure 3 right). The reconstructed trajectory contains all information on changes of direction as well as places along the way. For those reference points that are reconstructed in decompression, their timestamp is calculated based on an assumed linear movement behavior between start and end point of a chunk. This reconstructed information is sufficient for most tasks, specifically navigation support and most query-by-location services.

In a nutshell, the decompression algorithm iterates through the sequence of chunks stored in the compressed trajectory. It returns a sequence of vertices of $V_{G}$ that are a geometric representation of the traveled path through the network. For each of these vertices, it calculates a timestamp. In more detail, beginning with the start vertex of a chunk the algorithm adds edges of $E_{G}$ to the reconstructed trajectory until the start vertex of the next chunk is reached. To this end, it uses different strategies to determine which edge is to be added; these strategies depend on the description used for chunking.

Chunks based on the egocentric direction relation straight are decompressed by adding edges that head in the direction straight as seen from the previous edge. The direction relations are defined in a qualitative direction model that maps angle deviations between two edges to a direction relation [25|. At the current vertex it is checked which outgoing edges are in relation straight to the incoming edge. Since compression generates unambiguous descriptions for egocentric directions, there will always be only one such edge. In principle, the same approach is taken for the other kinds of descriptions. However, there might be more than one edge at a vertex that adheres to the same concept (identical labels, e.g., change from street $g$ to street $B$ in Figure 31. Therefore, a recursive function tries all candidates, i.e., searches a path from the current vertex to the final vertex in which all vertices are described by the used concept. At each vertex, only the edges that might fit at all are tested, i.e., the function excludes the incoming edge (to avoid going back) and all edges that cannot be described by the concept at hand. Thus, even though the recursive function 
has exponential complexity in theory, in practice the branching factor hardly ever exceeds 2. Figure 5 illustrates decompression based on the first two reference points stored in the compressed trajectory of Figure 3

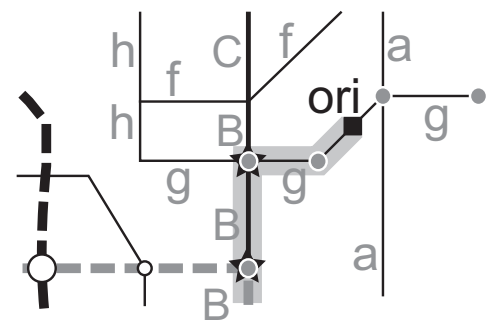

Trajectory:

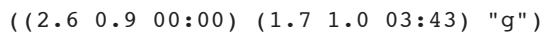

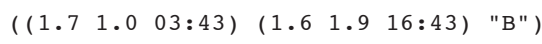

a)

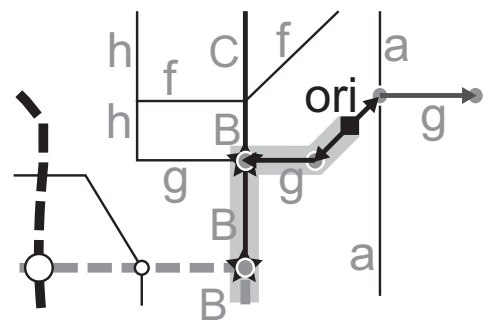

$\left.1: \quad\left(\begin{array}{llll}2.6 & 0.9\end{array}\right)\left(\begin{array}{lll}2.1 & 1.0\end{array}\right)\left(\begin{array}{lll}1.7 & 1.0\end{array}\right) \mathrm{gg}\right) \rightarrow \mathrm{B}$

$\left.2:\left(\begin{array}{lllll}(2.6 & 0.9\end{array}\right)\left(\begin{array}{lll}2.7 & 0.8\end{array}\right)\left(\begin{array}{lll}3.2 & 0.8\end{array}\right) \quad \mathrm{gl}\right)$

c)
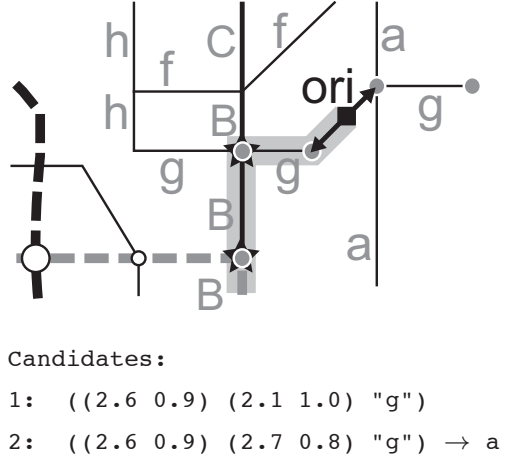

b)

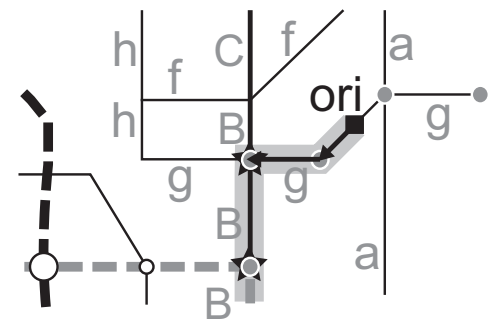

$\left.\left(\begin{array}{lllll}(2.6 & 0.9\end{array}\right)\left(\begin{array}{llll}2.1 & 1.0\end{array}\right)\left(\begin{array}{lll}1.7 & 1.0\end{array}\right) \quad \mathrm{g} "\right)$

d)

Figure 5: Part of the compressed trajectory of Figure 3 (a). Decompression starts at origin ori and explores both directions along street $g$, spanning two alternatives 1 and 2 (b). Alternative 2 meets street $a$ in the first decompression step (b), alternative 1 meets street $B$ in the second step (c). According to the sequence of stored reference points (see a), alternative 1 is the correct decompression and is, thus, maintained (d). Note that times are not given in (b), (c), (d) for readability reasons.

Algorithm 2 summarizes the decompression. Decompression needs as input both the compressed trajectory and the map against which compression has been performed.

\section{Evaluation}

STC has been evaluated using real tracking data and simulated movement data. Both illustrate the power of the approach for reducing the information that needs to be stored. 


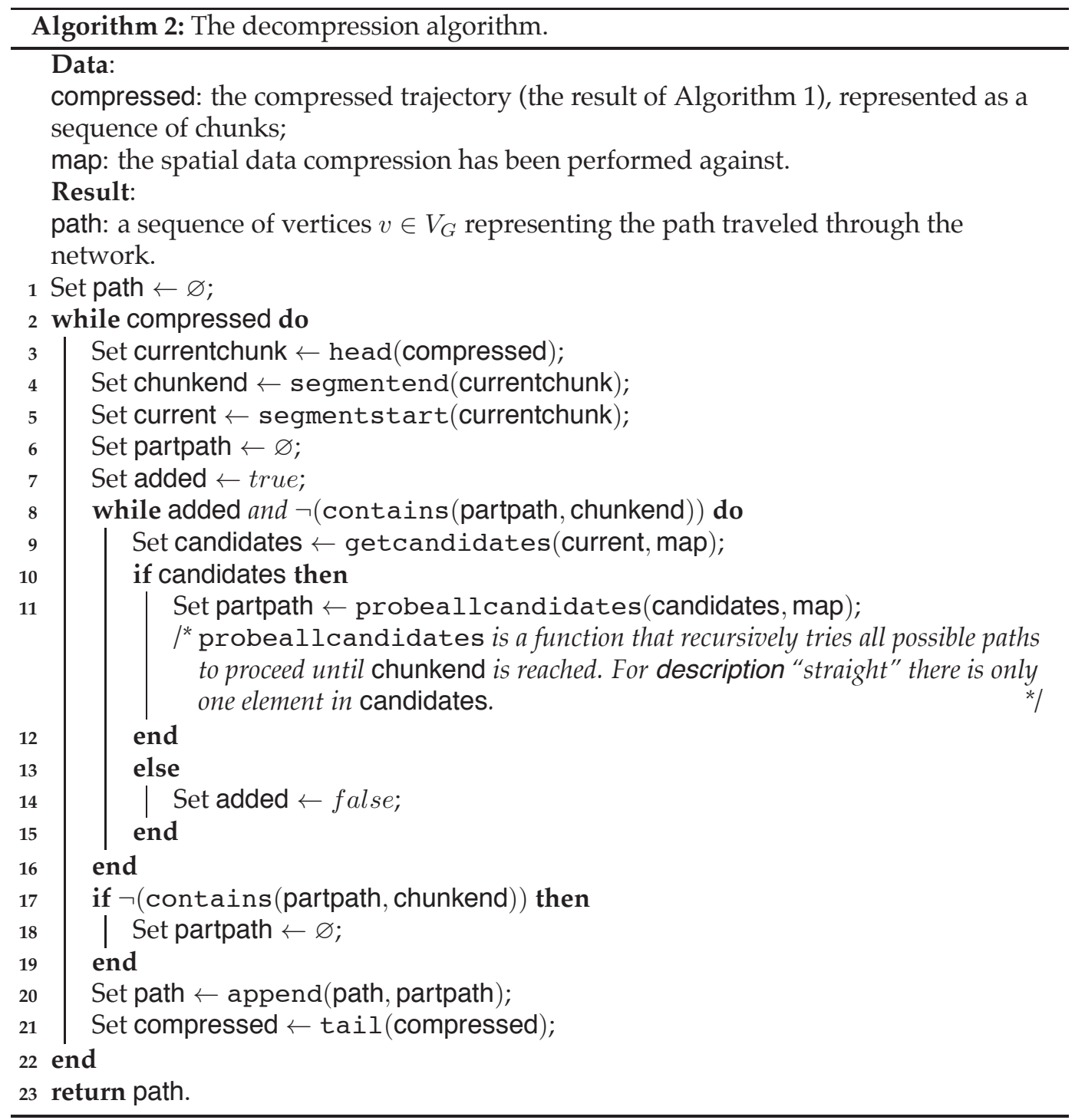

\subsection{Real tracking data}

18 everyday trajectories reflecting movement between regularly visited places of four persons have been recorded in the city of Bremen with a Garmin eTrex GPS device (sampling rate of 1 fix in 10 seconds, positional accuracy varies between 7 to 110 meters; the latter likely indicating positioning errors). The path lengths range from 980 to 7582 meters. Different transport modalities were used; the predominant one was traveling by bicycle.

Due to limitations of the used GPS tracking procedure (the fluctuant positional accuracy) and the subsequent difficulties in correct link identification, the trajectory data had to be preprocessed. The STC compression algorithm uses a trajectory matched to network data as input; in the evaluation, manual correction of wrong matches is sufficient to demon- 


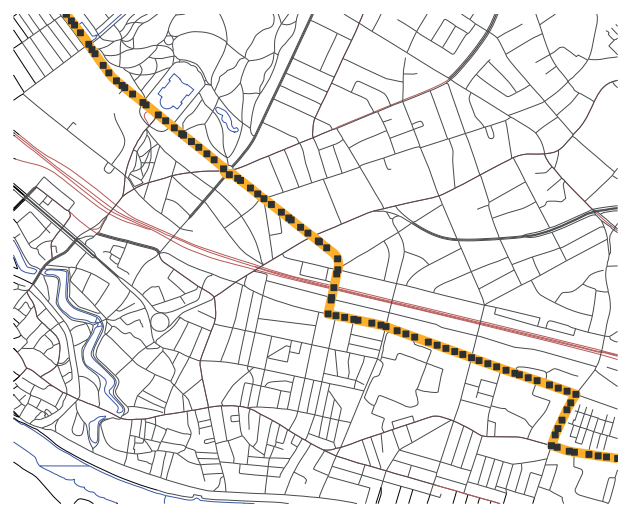

(a)

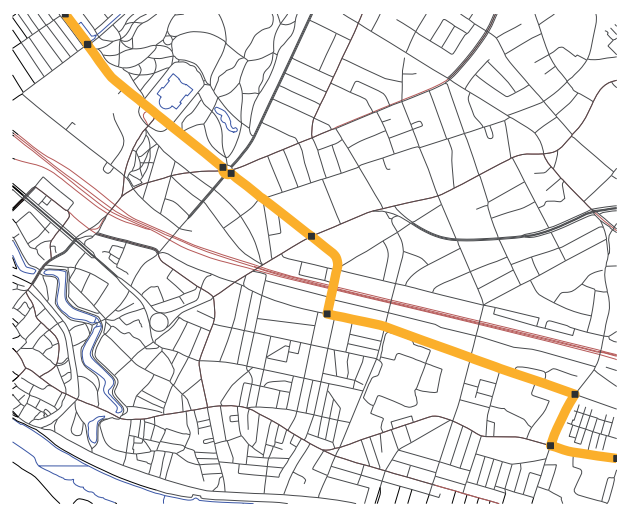

(b)

Figure 6: The map shows a part of Bremen where tests have been performed in. As an example, one of the 18 real world trajectories is shown (trajectory \#9 of Table 2). The Figure shows in (a) the original trajectory (bold line). The dots represent the fixes mapped to the street network; and in (b) the dots represent the reference points stored in the compressed trajectory.

strate the compression approach itself. In applications dealing with large data sets or realtime map matching (e.g., car navigation systems), the cleaning and map-matching of the data would need to be performed by more sophisticated algorithms as they have been presented in Section 2

The trajectories were matched onto the map $\left(V_{G}, E_{G}\right)$ using a simple point-to-point map matcher. This kind of matcher maps a GPS fix to the closest geometric or topological control point of the curve representing the street [46]. Simple point-to-point matchers are prone to matching errors as they assign the fix to the closest control point in its vicinity without any other plausibility check, such as topological constraint checking. Accordingly, the matching process produced some wrongly assigned fixes. Obviously wrong matches were corrected manually and additional synthetic fixes were added where link identification ambiguities occurred. This situation can occur when the satellite reception of the GPS device is limited and fixes with high positional inaccuracy are obtained. With a series of highly inaccurate fixes is it not possible to determine the exact traveled path; the implementation of reconstruction heuristics is required. TrackR [36|, a trajectory visualization software package, was used to this end. Linear spatiotemporal interpolation determined the synthetic fixes. This assumes constant velocity and straight movement along edges. It did not introduce or alter any information beyond resolving matching problems, an issue not in the focus of this paper.

STC has been tested on all recorded trajectories. Figure 6 shows an example. The trajectory is $4336 \mathrm{~m}$ long and comprises 86 fixes; the corresponding path in the map has 49 nodes (elements of $V_{T}$ ) and 102 coordinates (elements of $V_{G}$ ). Performing STC yields the 9 items of Table 1 . The table lists the descriptions of each item in plain text to better illustrate references of semantically meaningful elements in the compressed trajectory. In actual storage of the compressed trajectories, these items should be encoded with unique IDs connected to a lookup table in order to make the byte size of a stored element independent of the length of the attached street name. Decompressing the compressed trajectory restores the 
path geometry of the trajectory as it had been mapped to $\left(V_{G}, E_{G}\right)$. It also keeps those timestamps that are explicitly stored in the compressed trajectory. Despite some minor differences in the restored coordinates (discussed below), the visual appearance of the decompressed trajectory is indistinguishable from the trajectory shown in Figure 6a and is, therefore, not shown.

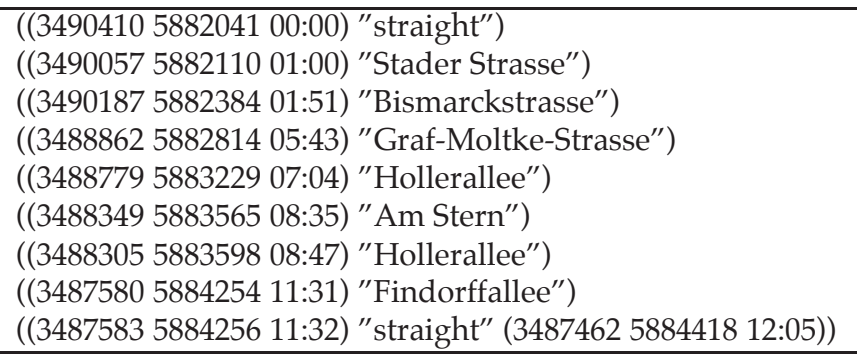

Table 1: An STC compressed trajectory.

Table 2 summarizes the results of compressing the 18 real world trajectories. The table lists the number of fixes of the original trajectory, nodes of $V_{T}$ after the mapping to the network, and items of the compressed trajectory (corresponding to those listed in Table 1). It also states two compression rates: The first compression rate ("compr(item)") looks at the number of items of the compressed trajectory ( 9 for the example of Table 1 ). The second compression rate ("compr(elem)") acknowledges that each item comprises two elements (a timestamped coordinate and a description) - except for the last item which additionally contains the timestamped coordinate of the destination. Thus, the 9 items of Table 1 correspond to 19 elements. The compression rates state the amount of data reduction, for example, a rate of 89.53 means that $89.53 \%$ less data needs to be stored compared to the number of fixes of the original trajectory.

The table also compares storage space of trajectory data. It states the file size in bytes for the original data of the mapped trajectories, the file size after compressing these files using zip-compression, and the file size of storing STC-compressed trajectories (both uncompressed and zip-compressed). These files are text files that simply store one node in each line for the mapped trajectories, respectively one item each line for the STC-compressed trajectories. The comparison with zip-compression allows for evaluating the performance of STC against a (general) lossless compression mechanism.

\subsection{Synthetic trajectories}

Alongside the real tracking data, the performance of STC was tested using synthetic trajectories from simulated movement. Using the same geographical data set that the real tracking data has been matched to, random paths were calculated on the combined network of streets and tracks. To get paths that are more plausible with respect to human movement through a network space, the approach to simplest paths |13| was used as a path search heuristic. This results in the spatial part of a trajectory; note that this already corresponds to a trajectory mapped to the network, no actual "unmapped" trajectory was generated. The temporal part was simulated by assuming movement with constant speed along the 


\begin{tabular}{|c|c|c|c|c|c|c|c|c|c|}
\hline \multirow[t]{2}{*}{ trajectory } & \multicolumn{5}{|c|}{ elements } & \multicolumn{4}{|c|}{ file size } \\
\hline & \#fixes & \#nodes & \#items & $\begin{array}{r}\text { compr } \\
\text { (item) }\end{array}$ & $\begin{array}{l}\text { compr } \\
\text { (elem) }\end{array}$ & traj. & $\begin{array}{l}\text { zip } \\
\text { traj. }\end{array}$ & STC & $\begin{array}{r}\text { zip } \\
\text { STC }\end{array}$ \\
\hline 1 & 77 & 20 & 6 & 92.21 & 83.12 & 7183 & 1388 & 222 & 294 \\
\hline 2 & 81 & 54 & 19 & 76.54 & 51.85 & 10222 & 1905 & 807 & 464 \\
\hline 3 & 95 & 58 & 10 & 89.47 & 77.89 & 8611 & 1671 & 440 & 377 \\
\hline 4 & 82 & 45 & 6 & 92.68 & 84.15 & 6415 & 1302 & 285 & 316 \\
\hline 5 & 152 & 103 & 24 & 84.21 & 67.76 & 15261 & 2834 & 945 & 515 \\
\hline 6 & 68 & 41 & 5 & 92.65 & 83.82 & 7180 & 1421 & 284 & 321 \\
\hline 7 & 103 & 71 & 12 & 88.35 & 75.73 & 9588 & 1892 & 401 & 365 \\
\hline 8 & 125 & 74 & 20 & 84.00 & 67.20 & 12920 & 2365 & 694 & 438 \\
\hline 9 & 86 & 49 & 9 & 89.53 & 77.90 & 6102 & 1274 & 339 & 332 \\
\hline 10 & 118 & 80 & 21 & 82.20 & 63.56 & 12280 & 2310 & 823 & 479 \\
\hline 11 & 20 & 13 & 5 & 75.00 & 45.00 & 1740 & 544 & 205 & 314 \\
\hline 12 & 42 & 42 & 8 & 80.95 & 59.52 & 6283 & 1266 & 342 & 352 \\
\hline 13 & 41 & 30 & 11 & 73.17 & 43.90 & 4534 & 1035 & 572 & 419 \\
\hline 14 & 83 & 46 & 13 & 84.34 & 67.47 & 7059 & 1470 & 444 & 424 \\
\hline 15 & 57 & 51 & 6 & 89.47 & 77.19 & 6814 & 1335 & 228 & 302 \\
\hline 16 & 56 & 29 & 10 & 82.14 & 62.50 & 4120 & 930 & 373 & 339 \\
\hline 17 & 90 & 43 & 10 & 88.89 & 76.67 & 5555 & 1190 & 401 & 363 \\
\hline 18 & 89 & 65 & 10 & 88.76 & 76.40 & 9139 & 1771 & 441 & 378 \\
\hline
\end{tabular}

Table 2: Results of the 18 real world trajectories. compr(item) states the compression rate when comparing items of the compressed trajectory; compr(elem) the compression rate for the number of stored elements. File size is given in bytes; "traj." and "zip traj." state file sizes for the (zipped) mapped trajectories, "STC" and "zip STC" for the (zipped) STC compressed trajectories.

path. The speed was randomly set to values between $6 \frac{\mathrm{m}}{\mathrm{s}}$ and $14 \frac{\mathrm{m}}{\mathrm{s}}$. A sampling rate of 1 fix every 5 seconds was used. Using the speed and sampling rate, the number of fixes an actual trajectory along the previously determined path would have was calculated. 1000 such trajectories were created. They vary in length between 1 and 497 fixes (mean: 139 fixes). Figure 7 illustrates the results of their STC compression. The 1000 trajectories are grouped into ten length classes (shortest $10 \%, 10 \%$ to $20 \%, \ldots$ ). As for the real tracking data, the number of nodes represents the number of elements of $V_{T}$, i.e., the number of topological nodes that describe the movement through the network after mapping the trajectory to the network.

\subsection{Results}

STC achieves a high compression rate. Looking at the example of Figure 6 again, instead of storing 86 fixes, only 9 items need to be stored. This corresponds to a compression rate of $89.53 \%$. Considering the number of elements the compression rate is still $77.90 \%$. As can be seen in Table 2. similar results are achieved for all real world trajectories (mean compression rate (items): $85.25 \%$, standard deviation: 6.02; mean compression rate (elements): $68.98 \%$, st.d.: 12.61). These findings are confirmed by the results of the synthetic trajectories test. STC significantly decreases the amount of data to be stored here as well. Comparing

www.josis.org 


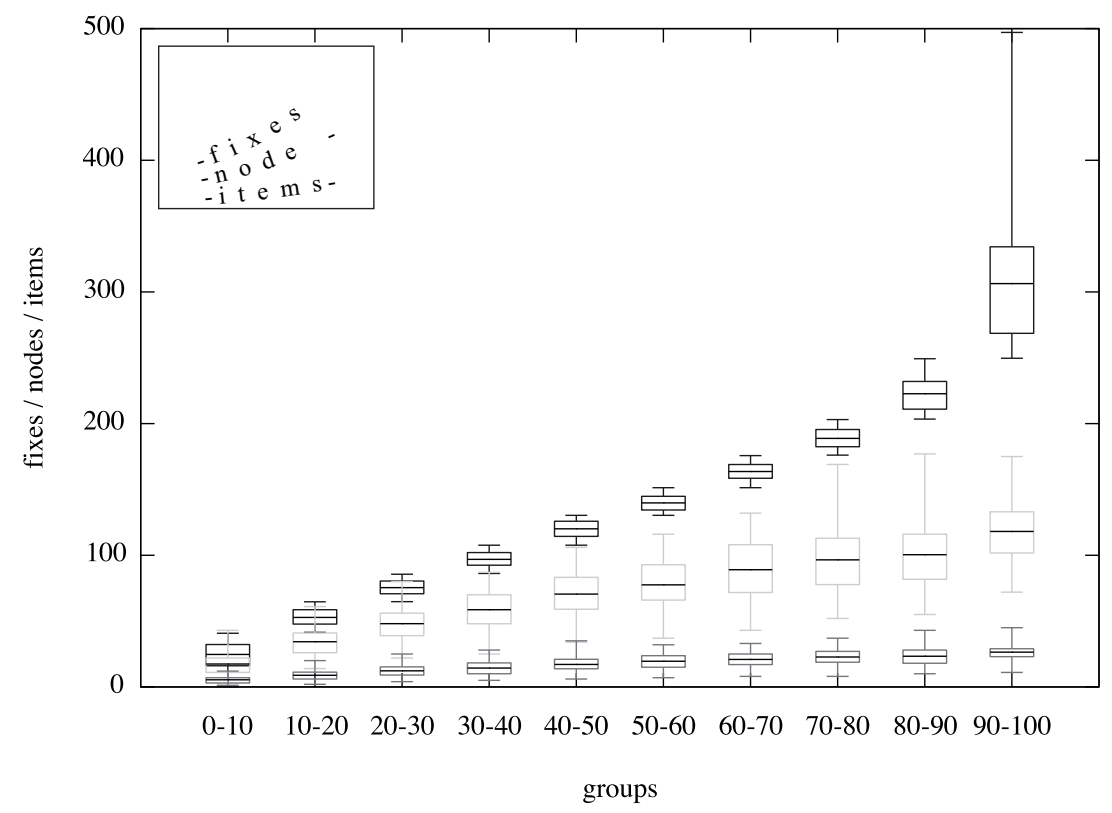

Figure 7: Results for synthetic trajectories. Shown are the number of fixes in the original trajectory, nodes in the corresponding path, items in the compressed trajectory. Trajectories have been divided into ten length classes according to the number of fixes (lowest $10 \%$, $10-20 \%, \ldots$ ). The box-whisker plot displays the minimum and maximum of a group (the Tends of the lines), the interquartile range (the boxes), and the mean number (the horizontal lines in the boxes).

the number of items in the compressed trajectories to the number of fixes, on average the compression rate for the first group is $77.9 \%$, for the last group $91.4 \%$. Accounting for the fact that two elements (a start reference point and a description) have to be stored in each item, the rates change to $51.8 \%$ and $82.4 \%$, respectively. This is still a large reduction.

Matching trajectories to the network already would allow for some compression of the data. In this case only the nodes of the corresponding graph that the matched trajectory passes through need to be stored, annotated with a time stamp of when they are passed. Such an approach, while being simplistic in its implementation, provides an upper bound for the compression rate of the approach by Cao and Wolfson [6]. Figure 7 also shows the compression rates achieved with this method. They range from an average of $29.50 \%$ for the first group to $61.44 \%$ for the last group. This compression is the result of the preprocessing step of STC. Performing chunking on the network path (represented by the nodes) by exploiting the semantic information attached to the network's elements significantly increases these compression rates. STC compression is between $20 \%$ to $30 \%$ higher compared to simple node compression for every group (average $26.76 \%$ ).

It can also be observed that with increasing number of fixes the compression rate increases. The longer the trajectories, the better the compression. For very short trajectories (those with 1 or 2 fixes only), STC actually creates overhead information as 1 fix needs to 
be represented using 3 elements (start and end reference point, description). However, the number of STC items increases much slower than the number of fixes. The average number of fixes in the first group is 24.67, in the last group 306.31 (factor 12). The average number of items in the first group is 5.45, for the last group it is 26.43 (factor 4.8).

All reconstructed reference points are annotated with estimated timestamps assuming constant speed within a chunk. In some cases decompression returns a path that partially differs from the original path (the trajectory's realization in $V_{G}, E_{G}$ ). For the example of Figure 6 the reconstructed path has 2 coordinates more than the original path. The underlying geographic data set has individual representations of different lanes for some streets, resulting in different geometric representations. The decompression algorithm may choose to navigate roundabouts clockwise or counterclockwise. These effects may cause differences in the geometric reconstruction of trajectories (see further discussion in Section 81. In the example the reconstruction of movement through the roundabout explains the geometric differences.

The results reported so far compare the number of elements that need to be stored for trajectories. When looking at file size, i.e., the actual space trajectories take up on storage media, STC also performs well. Zip compressing trajectories certainly results in a significant reduction of storage space-on average a reduction of $80.65 \%$ for the 18 trajectories of Table 2. However, STC-compressing these trajectories results in an average reduction of $96.04 \%$. Reducing trajectories to their semantic core, thus, clearly provides a more powerful compression than a simple (syntactic) reduction of the raw data. In fact, it is such a good compression that zip-compressing the STC-compressed trajectories on average results in an increase of storage space again (negative average reduction of $-9.07 \%$ ).

\section{Discussion}

There are four main insights that can be drawn from the evaluation: 1) STC compresses trajectories to only a fraction of raw data volumes; 2 ) it also drastically reduces the amount of data to be stored compared to a trajectory's network representation; 3) for purposeful movement through a transport network, with increasing length of trajectories, compression increases; the longer a trajectory is, the better is the compression rate; 4) STC reconstructs compressed trajectories featuring all essential information. Highway travel may further illustrate the power of STC. The only information that needs to be stored is the position of ramps on and off the highway and the highway's name (e.g., a trip on the German interstate A7 from Hannover to Ulm of about 550km, or 6 hours, ideally could be stored using only two information items).

Increasing compression rates with increasing length of trajectories can be explained with the number of direction changes along a path, i.e., those turns at an intersection that are not classified as "straight." For the tested trajectories, the ratio between the number of nodes in the path corresponding to the trajectory and the number of direction changes is essentially constant over all groups of Figure 7 (between 3.4 and 3.89, no monotone increase). Even though with an increasing number of nodes (longer trajectories) the options for turning somewhere increase, longer trajectories representing purposeful movement in a network contain more and longer segments that are chunkable. This can be attributed to the common three-part division of traveling in urban networks [10|: 1) getting from origin on to the system of main streets; 2) traveling along main streets (where only few direction 
changes occur); 3) near the end, getting off the main streets again to move to the destination. Due to the chosen path search heuristics in the evaluation, this pattern occurs in the data despite it being random paths.

As explained above, ambiguity may arise in decompressing trajectories. Since label compression does not include heading information, in some situations there may be more than one possible path from start position to end position of a chunk. In such situations, decompression chooses the first valid path it finds. This is due to the fact that STC so far ignores movement modality and associated traffic regulations. Means to account for these are part of future work.

In general, there is a direct relationship between minimizing the stored data in compression and an increased effort in restoring the trajectory again. Since only the essential reference points for describing motion in the network are stored, intermediate edges need to be recalculated, which involves resolving possible ambiguities. At the other end of this spectrum every edge that is traversed by the path could be stored, which would take up more space, but would only require an ID lookup. It is also possible to extend the data stored in the tuple representing the chunk. Along with the description element (e.g., street name) heading information could be captured; with every change of heading a new chunk would be created. Such a compression would combine at least some reference points and, thus, would be a more compact representation than storing all edge IDs. Decompression then could select that edge at a vertex that is labeled by the used description and heads in the correct direction. However, this paper aims at demonstrating the maximally possible data reduction; a detailed evaluation of the relation between data compression and decompression effort is part of future work. Something to note in this context is that even though decompression has exponential run time in theory, in practical terms it runs faster than compression. Due to the low branching factor of the network (street networks are to a most part planar graphs) run time is nearly linear. This is desirable behavior, since in most applications, a trajectory is compressed only once for data storage but may be decompressed several times for further computation.

A specific property of the STC approach is the dependency on having available the same data set for decompression as was used for compression. In contrast to image compression procedures, for example, that rely on a "lightweight" compression algorithm, STC relies on the "heavyweight" input of the (same) semantic data. This is no problem within a single application that either runs on a single machine or is client-server based. And with today's advances in ubiquitously available spatial data accessible from the web, sharing compressed trajectories across applications and people becomes increasingly easier. It can be expected that in near future (quasi-)standard spatial data emerges that is used in many applications. But even when using a different data set for decompression than for compression, the traveled path through the network can likely be reconstructed. Compression is based on semantic information, such as street names or tram lines, and abstracts geometry to a qualitative level by using direction relations that correspond to angle intervals. If it is possible to anchor the compressed trajectory in the new data set, i.e., to determine position of origin and initial heading, recovery of the trajectory will be possible using the same methods as described above. Only if the data set for decompression contains less information than the compression set, i.e., if specific layers of information that were used in compression are missing or are incomplete, reconstruction fails. Still, if at least parts of the path can be recovered, it may be possible to use heuristics based on human wayfinding 
behavior (e.g., principles identified by [17|) for determining the most probable trajectory to fill in the gaps.

Another limitation is the dependency on map-matching as a pre-processing step. Map matching algorithms always try to converge toward infrastructural elements. However, humans are not tied to moving exactly along officially mapped streets, they may take shortcuts, like walking straight across a lawn instead of walking around it. A map matching algorithm will try to match the fixes to the paths surrounding the lawn. This introduces some error compared to the original movement. Thus, the quality of STC relies on the quality of the applied map matching. Finally, one may argue that reconstructing a trajectory with only interpolated timestamps and not the original trajectory with all its timestamps does not really mirror a full compression-decompression process. However, as previously argued, the reconstructed trajectory represents the semantically meaningful aspects of the movement behavior. Many fixes, on the other hand, can be expected to be just noise in the movement description that originate from inaccurate localization.

\section{Applications of STC trajectories}

A major advantage of STC is that the compressed trajectories are human-readable. STC not only compresses streams of fixes, but it also transforms raw data into a semantically enriched summary of the movement episodes, not unlike a narrative summary. This opens opportunities for applying STC in a navigation context; trajectories compressed with STC can be used for personalizing mapping and navigation services. For example, personalized navigation assistance as proposed in [37| requires a user model that consists of previously traveled paths and visited places. As STC-trajectories consist of these elements, personalized assistance can be directly generated from them.

Thinking this further, the semantic representation of trajectories is ideally suited to compare movement within and between different people or objects. For example, checking movement of a specific person in a network for consistency becomes easy, as any major deviation would emerge as differences in the STC compressed representation, while minor (irrelevant) deviations would be filtered out in the compression and, accordingly, would not need to be dealt with. Such comparisons have their applications in areas such as navigation services (as discussed above), logistics, or surveillance. Furthermore, it also becomes easily possible to check movement behavior of different people and objects for similarities, for example, to identify people that have similar travel patterns. This has practical application in areas such as intelligent transport (e.g., identifying opportunities for ride sharing), spatial profile matching, or route optimization in logistics.

Such applications will require querying STC trajectories. Given that STC compresses trajectory data that represents movement in a network environment, there are some differences to the approaches discussed in Section 2

Trajectory queries STC is all about retaining the relevant semantic information of trajectories while reducing redundancies. Since the network elements that trajectories move along are restored in decompression, any queries related to a trajectory's shape will result in the same results as if querying the original map-matched trajectory. This holds, for example, for queries, such as: "Where did object $x$ stop?" "Which trajectories travel in a north-south direction?" "Did object $x$ move along network element $y$ ?" The first two 
queries can even be answered on the compressed trajectory without a need to decompress again. Also, simple similarity queries can be answered directly using STC trajectories (including queries, such as "Which other objects follow a similar path to this one?" or "What other objects pass through this network element?"). STC compresses a trajectory into a sequence representation; therefore, similarity techniques based on quantifying the difference between sequences (e.g., edit distance) present a natural fit for similarity queries on STC data $[9,11 \mid$.

Network queries Papadias et al. [31| reformulated queries that are typically run on moving object databases to account for movement in networks. These queries are $(k)$ nearest neighbor, range, and closest-pair queries. All the queries use network distance instead of Euclidean distance. In order to answer these queries, STC trajectories need to be decompressed, except if a query is run using any of the start or end points of any of the chunks as query point $q$.

STC trajectories will perform well for any queries that do not require high precision. Queries on STC trajectories are precise to at least the level of edges (as edges are the basic elements of the compression algorithm). Queries, such as "When did object $x$ pass edge $y$ ?" will deliver useful results since the "objects" moving along the edges usually do not have random speed or acceleration/deceleration patterns, but exhibit consistent patterns. Trains and trams normally move with similar speed patterns, and likewise cars are bound by traffic regulations. However, the STC approach fails to pick up extreme behavior. For example, if an object accelerates and decelerates rapidly between start and end points of chunks, this movement behavior would be impossible to represent and query in a STC trajectory.

Network queries lead to correct results on the level of granularity of network distance from an edge. However, distance from edge is a more complex operation, with a less clear semantics, than distance from point. Thus, usually these queries will be run using an interpolated point $q_{i}$ on that edge. Interpolated fixes do not need to match with fixes in the original, matched trajectory; this may result in deviations between query results on the original trajectory and the STC trajectory. For example, if two or more objects of the requested type have a similar distance to the original point $q_{o}$ (often, this corresponds to a high density in distribution of that object type), a query using the interpolated point $q_{i}$ may return the object that is in fact farer away from $q_{o}$ if the interpolation moves $q_{i}$ closer to that object. Likewise, range queries using $q_{i}$ may miss some objects on or close to the fringe of the selected range as seen from $q_{o}$, or include some that are just missed by a query using $q_{o}$.

To sum up, STC trajectories perform well for any queries related to trajectory shape and similarity. They will also generally perform well for any queries that do not require very high precision, i.e., where results are expected to be on a granularity level corresponding to edges. These are the kind of queries required for the application areas discussed above.

\section{Conclusions and outlook}

This paper presents semantic trajectory compression (STC) for compressing large volumes of trajectory data. STC exploits the semantic embedding of movement in a geographical context. It matches individual trajectories to a semantically annotated map and aggregates movement chunks based on identical semantic descriptions. STC extends concepts of 
network-constrained indexing in moving object databases and techniques used in wayfinding assistance. This paper's main contribution lies in illustrating that the embedding of human movement in its geographic context (here an urban transport network) can be exploited for compressing large volumes of raw trajectory data. Initial experiments show that semantic compression achieves high compression rates for real and synthetic trajectories, with arguably acceptable information loss.

As part of future work, movement modality ("walking," "cycling," "driving," "going by tram") will be explored to improve compression and decompression in STC (cf. [14 41|). Knowing the movement modality allows for an improved identification of reference points. For example, for an individual traveling by train in compression intermediate reference points, where the train stopped at stations, can be ignored and only the positions of the stations where the individual entered and left the train need to be stored as reference points. In decompression, movement modality reduces ambiguity. Traffic regulations restrict the possible edges that movement may have occurred on, dependent on the modality. This would rule out going clockwise through a roundabout when driving a car, for example. Such possible behavior cannot be excluded for pedestrian movement (and may also happen in motorized traffic; see the analysis in [44|). Further ambiguities may be solved by using heuristics accounting for the likelihood for a correct match, for example, based on the travel speed (cf. [28], who use odometry data to exclude unlikely candidates in matching GPS fixes to a network).

So far, STC was developed primarily with a data compression motivation. Still, some potential application areas of STC-compressed trajectories were suggested. These applications require queries on STC trajectories; their general behavior was discussed. However, querying STC-compressed trajectories demands further analysis and, accordingly, alternative evaluation techniques for STC will be developed. The experiments in Section 5 investigated STC with respect to data compression rate and spatial accuracy, respectively data loss of the compression-decompression sequence. Additional experiments will explicitly evaluate the spatiotemporal restoration accuracy in a more rigorous way, for example "Where in space and time lies a fix of the decompressed trajectory with respect to its counterpart in the original uncompressed trajectory?" (cf., e.g., [15] who present an error analysis for time slice queries). One way of assessing this spatiotemporal restoration accuracy is the stochastic analysis of the distance between randomly selected pairs of corresponding fixes (decompressed versus original). The compression of a trajectory could be called "spatiotemporally accurate," if the average spatiotemporal distance between such pairs does not exceed a given threshold. Such evaluation, however, requires more accurate GPS tracks than were available for the present initial study.

\section{Acknowledgments}

Support by the German Science Foundation (DFG) and Group of Eight/DAAD Australia Germany Joint Research Co-operation Scheme is acknowledged.

\section{References}

[1] Agarwal, P. K., Guibas, L. J., Edelsbrunner, H., Erickson, J., Isard, M., Har-Peled, S., Hershberger, J., Jensen, C., Kavraki, L., Koehl, P., Lin, 
M., Manocha, D., Metaxas, D., Mirtich, B., Mount, D., Muthukrishnan, S., PAi, D., SAcks, E., SnOeYinK, J., Suri, S., AND Wolfson, O. Algorithmic issues in modeling motion. ACM Computing Surveys 34, 4 (2002), 550-572. doi:10.1145\%2F592642.592647

[2] Alvares, L. O., Bogorny, V., Kuijpers, B., Fernandes de Macedo, J. A., MoeLANS, B., AND VAISMAN, A. A model for enriching trajectories with semantic geographical information. In Proc. 15th Annual ACM International Symposium on Advances in GIS (ACMGIS) (New York, 2007), ACM, pp. 1-8. doi:10.1145/1341012.1341041.

[3] AshBrooK, D., AND STARNER, T. Using GPS to learn significant locations and predict movement across multiple users. Personal Ubiquitous Computing 7, 5 (2003), 275-286. doi:10.1007/ s00779-003-0240-0

[4] Bernstein, D., And Kornhauser, A. An introduction to map matching for personal navigation assistants. Tech. rep., New Jersey TIDE Center, 1996.

[5] Brakatsoulas, S., Pfoser, D., Salas, R., And WenK, C. On map-matching vehicle tracking data. In Proc. 31st International Conference on Very Large Databases (VLDB) (2005), VLDB Endowment, pp. 853-864.

[6] CAO, H., AND WOlfSON, O. Nonmaterialized motion information in transport networks. In Database Theory (ICDT) (Berlin, 2005), T. Eiter and L. Libkin, Eds., vol. 3363 of Lecture Notes in Computer Science, Springer, pp. 173-188. doi:10.1007/978-3-540-30570-5_12

[7] CaO, H., Wolfson, O., And Trajcevski, G. Spatio-temporal data reduction with deterministic error bounds. VLDB Journal 15, 3 (2006), 211-228. doi:10.1007/ s00778-005-0163-7

[8] Castelli, G., Mamei, M., And Rosi, A. The whereabouts diary. In Location- and Context-Awareness, J. Hightower, B. Schiele, and T. Strang, Eds., vol. 4718 of Lecture Notes in Computer Science. Springer, Berlin, 2007, pp. 175-192. doi:10.1007/978-3-540-75160-1

[9] Chen, L., Özsu, M. T., AND ORIA, V. Symbolic representation and retrieval of moving object trajectories. In Proc. 6th ACM SIGMM International Workshop on Multimedia Information Retrieval (New York, 2004), MIR '04, ACM, pp. 227-234. doi:10.1145/1026711.1026749.

[10] DALE, R., Geldof, S., AND PROST, J.-P. Using natural language generation in automatic route description. Journal of Research and Practice in Information Technology 37, 1 (2005), 89-105.

[11] Dodge, S., Weibel, R., AND Forootan, E. Revealing the physics of movement: Comparing the similarity of movement characteristics of different types of moving objects. Computers, Environment and Urban Systems 33, 6 (2009), 419-434. doi:10.1016/j.compenvurbsys.2009.07.008

[12] Douglas, D. H., AND PeuckeR, T. K. Algorithms for the reduction of the number of points required to represent a digitized line or its caricature. Cartographica 10, 2 (1973), 112-122. doi:10.3138/ FM57-6770-U75U-7727. 
[13] DucKham, M., AND KuliK, L. "Simplest" paths: Automated route selection for navigation. In Spatial Information Theory (Berlin, 2003), W. Kuhn, M. Worboys, and S. Timpf, Eds., vol. 2825 of Lecture Notes in Computer Science, Springer, pp. 169-185. doi:10.1007/978-3-540-39923-0_12

[14] DyKeS, J. A., AND MounTAIn, D. M. Seeking structure in records of spatio-temporal behaviour: Visualization issues, efforts and application. Computational Statistics and Data Analysis 43, 4 (2003), 581-603. doi:10.1016/S0167-9473(02)00294-3

[15] Frentzos, E., AND TheOdoridis, Y. On the effect of trajectory compression in spatiotemporal querying. In Advances in Databases and Information Systems (Berlin, 2007), Y. Ioannidis, B. Novikov, and B. Rachev, Eds., vol. 4690 of Lecture Notes in Computer Science, Springer, pp. 217-233. doi:10.1007/978-3-540-75185-4_17

[16] Galton, A. Dynamic collectives and their collective dynamics. In Spatial Information Theory (Berlin, 2005), A. Cohn and D. M. Mark, Eds., vol. 3693 of Lecture Notes in Computer Science, Springer, pp. 300-315. doi:10.1016/S0167-9473(02)00294-3.

[17] Golledge, R. G. Path selection and route preference in human navigation: A progress report. In Spatial Information Theory (Berlin, 1995), A. U. Frank and W. Kuhn, Eds., vol. 988 of Lecture Notes in Computer Science, Springer, pp. 207-222.

[18] GReenfield, J. Matching GPS observations to locations on a digital map. In Proc. 81st Annual Meeting of the Transportation Research Board (2002).

[19] Gudmundsson, J., Katajainen, J., Merrick, D., Ong, C., And Wolle, T. Compressing spatio-temporal trajectories. Computational Geometry-Theory and Applications 42, 9 (2009), 825-841. doi:10.1016/j.comgeo.2009.02.002

[20] Gudmundsson, J., van Kreveld, M., And Speckmann, B. Efficient detection of patterns in 2D trajectories of moving points. GeoInformatica 11, 2 (2007), 195-215. doi:10.1007/ s10707-006-0002-z

[21] GÜting, H., DE AlmeidA, T., AND DinG, Z. Modeling and querying moving objects in networks. The VLDB Journal 15 (June 2006), 165-190. doi:10.1007 / s00778-005-0152-x

[22] GÜting, R. H., And SchneIder, M. Moving Objects Databases. Morgan Kaufmann, San Francisco, 2005.

[23] Kang, J. H., Welbourne, W., Stewart, B., And Borriello, G. Extracting places from traces of locations. SIGMOBILE Mobile Computing and Communications Review 9, 3 (2005), 58-68. doi:10.1145/1094549.1094558

[24] Klippel, A., Hansen, S., Richter, K.-F., And Winter, S. Urban granularities-A data structure for cognitively ergonomic route directions. GeoInformatica 13, 2 (2009), 223-247. doi:10.1007/s10707-008-0051-6

[25] Klippel, A., AND Montello, D. R. Linguistic and nonlinguistic turn direction concepts. In Spatial Information Theory (Berlin, 2007), S. Winter, M. Duckham, L. Kulik, and B. Kuipers, Eds., vol. 5266 of Lecture Notes in Computer Science, Springer, pp. 354-372. doi:10.1007/978-3-540-74788-822

WwW.josis.org 
[26] Klippel, A., TAppe, H., AND Habel, C. Pictorial representations of routes: Chunking route segments during comprehension. In Spatial Cognition III (Berlin, 2003), C. Freksa, W. Brauer, C. Habel, and K. F. Wender, Eds., vol. 2685 of Lecture Notes in Artificial Intelligence, Springer, pp. 11-33. doi:10.1007/3-540-45004-12.

[27] LI, X., AND LiN, H. Indexing network-constrained trajectories for connectivity-based queries. International Journal of Geographical Information Science 20, 3 (2006), 303-328. doi:10.1080/13658810500432570

[28] Li, X., ZhanG, X., AND LiN, H. Deriving network-constrained trajectories from sporadic tracking points collected in location-based services. Geo-Spatial Information Science 12, 2 (2009), 85-94. doi:10.1007/ s11806-009-0232-y.

[29] LiaO, L., FOX, D., AND KAUTZ, H. Extracting places and activities from GPS traces using hierarchical conditional random fields. International Journal of Robotics Research 26, 1 (2007), 119-134. doi:10.1177/ 0278364907073775

[30] Meratnia, N., AND DE BY, R. A. Spatiotemporal compression techniques for moving point objects. In Advances in Database Technology, EDBT 2004 (Berlin, 2004), E. Bertino, S. Christodoulakis, D. Plexousakis, V. Christophides, M. Koubarakis, K. Bohm, and E. Ferrari, Eds., vol. 2992 of Lecture Notes in Computer Science, Springer, pp. 765-782. 10.1007/978-3-540-24741-8_44

[31] Papadias, D., Zhang, J., Mamoulis, N., And TaO, Y. Query processing in spatial network databases. In Proc. 29th International Conference on Very Large Databases (VLDB) (2003), VLDB '2003, VLDB Endowment, pp. 802-813. doi:10.1016/B978-012722442-8/50076-8

[32] Potamias, M., Patroumpas, K., And Sellis, T. Sampling trajectory streams with spatiotemporal criteria. In Proc. 18th International Conference on Scientific and Statistical Database Management (SSDBM) (Washington, DC, 2006), IEEE Computer Society, pp. 275-284. doi:10.1109/SSDBM.2006.45

[33] Quddus, M. A., Ochieng, W. Y., And Noland, R. B. Current map-matching algorithms for transport applications: State-of-the art and future research directions. Transportation Research Part C: Emerging Technologies 15, 5 (2007), 312-328. doi:10.1016/j.trc.2007.05.002

[34] Quddus, M. A., Ochieng, W. Y., ZhaO, L., And Noland, R. B. A general map matching algorithm for transport telematics applications. GPS Solutions 7, 3 (2000), 157-167. doi:10.1007/s10291-003-0069-z

[35] Richter, K.-F. Context-Specific Route Directions-Generation of Cognitively Motivated Wayfinding Instructions, vol. 314 of DisKI. IOS Press, Amsterdam, 2008.

[36] SCHMID, F. Formulating, identifying, and analyzing individual spatial knowledge. In Seventh IEEE International Conference on Data Mining Workshops (ICDMW 2007) (Los Alamitos, CA, 2007), IEEE Computer Society.

[37] SCHMID, F. Knowledge based wayfinding maps for small display cartography. Journal of Location Based Services 2, 1 (2008), 57-83. doi:10.1080/17489720802279544. 
[38] SCHMid, F., AND RichteR, K.-F. Extracting places from location data streams. In Second International Workshop on Ubiquitous Geographical Information Services (UbiGIS) (2006).

[39] Schmid, F., Richter, K.-F., AND Laube, P. Semantic trajectory compression. In Advances in Spatial and Temporal Databases (Berlin, 2009), N. Mamoulis, T. Seidl, T. Pedersen, K. Torp, and I. Assent, Eds., vol. 5644 of Lecture Notes in Computer Science, Springer, pp. 411-416. doi:10.1007/978-3-642-02982-0_30

[40] Sistla, A. P., Wolfson, O., Chamberlain, S., And DaO, S. Modeling and querying moving objects. In 13th International Conference on Data Engineering (ICDE13) (1997).

[41] Sмүтн, C. S. Mining mobile trajectories. In Geographic data mining and knowledge discovery, H. J. Miller and J. Han, Eds. Taylor and Francis, London, UK, 2001, pp. 337361. doi:10.4324/9780203468029_chapter_14

[42] Spaccapietra, S., Parent, C., Damiani, M. L., De Macedo, J. A., Portoa, F., AND VANGENOT, C. A conceptual view on trajectories. Data and Knowledge Engineering 65, 1 (2008), 126-146. doi:10.1016/j.datak.2007.10.008

[43] Tiesyte, D., And Jensen, C. S. Similarity-based prediction of travel times for vehicles traveling on known routes. In Proc. 16th ACM SIGSPATIAL International Conference on Advances in Geographic Information Systems (ACMGIS) (New York, 2008), ACM, pp. 1-10. doi:10.1145/1463434.1463452

[44] TURNER, A. The role of angularity in route choice. In Spatial Information Theory (Berlin, 2009), K. S. Hornsby, C. Claramunt, M. Denis, and G. Ligozat, Eds., vol. 5756 of Lecture Notes in Computer Science, Springer, pp. 489-504. doi:10.1007/978-3-642-03832-7_30.

[45] TVersky, B., AND LeE, P. U. How space structures language. In Spatial Cognition, C. Freksa, C. Habel, and K. F. Wender, Eds., vol. 1404 of Lecture Notes in Artificial Intelligence. Springer, Berlin, 1998, pp. 157-175. doi:10.1007/3-540-69342-4_8

[46] White, C. E., Bernstein, D., And Kornhauser, A. L. Some map matching algorithms for personal navigation assistants. Transportation Research Part C: Emerging Technologies 8, 1-6 (2000), 91-108. doi:10.1016/S0968-090X(00)00026-7.

[47] Yan, Z., Macedo, J., Parent, C., And Spaccapietra, S. Trajectory ontologies and queries. Transactions in GIS 12 (2008), 75-91. doi:10.1111/j.1467-9671.2008.01137.x 